\title{
PERCEPCIÓN DE MALTRATO AL PROFESIONAL DE SALUD DURANTE LA ESPECIALIZACIÓN - RESIDENCIA MÉDICA
}

\section{PERCEPTION OF MALTRATION TO THE HEALTH PROFESSIONAL DURING SPECIALIZATION - MEDICAL RESIDENCE}

Javier Rubén Tovar Brandán¹, Manul Yampufe Cornetero², Teresa Luisa pareja Pera³, Christian R. Miranda Orillo

\section{RESUMEN}

Entre los múltiples retos que enfrenta un interno, un residente se encuentran las relaciones intrahospitalarias. En estas relaciones se han identificado maltratos hacia el interno, médico residente que interfieren con su etapa formativa que trae consecuencia a futura al propio médico, si no se traslada al Acto Médico y es en la atención en la Seguridad de Paciente. Sin embargo, se conoce poco o nada sobre este fenómeno en Perú.

Palabras clave: Maltrato; Residencias Médicas; Abusos; Depresión; Seguridad del Paciente.

\begin{abstract}
Among the many challenges faced by a resident are intra-hospital relations. In these relationships have been identified abuse to the resident doctor that interfere with their formative stage that brings future consequences to the doctor himself if not moved into care in Patient Safety. However, little or nothing is known about this phenomenon in Peru.
\end{abstract}

Key words: Abuse; Medical Residences; Abuses; Depression; Patient safety.

\footnotetext{
${ }^{1}$ Docente Principal de la Facultad de Medicina Humana, Universidad Nacional Mayor de San Marcos. Maestro en Administración Estratégica y Derecho Sanitario y Bioética.

${ }^{2}$ Docente Principal de la Facultad de Medicina Humana, Universidad Nacional Mayor de San Marcos. Magíster en Fisiología

${ }^{3}$ Docente Auxiliar de la Facultad de Medicina Humana Universidad Nacional Mayor de San Marcos. Magíster en Salud Publica.

${ }^{4}$ Coordinador de Residentado Medico Hospital Nacional Guillermo Almenara Irigoyen.
} 
Tabla 1: Datos tomados de temperatura y densidad del aceite lubricante Shell Helix HX7 10W-40

\begin{tabular}{cc}
\hline $\mathbf{T}$ & $\begin{array}{c}\mathbf{D} \\
\left({ }^{\circ} \mathbf{C}\right)\end{array}$ \\
\hline 45.00 & 848 \\
42.00 & 850 \\
39.50 & 851 \\
37.00 & 852 \\
36.00 & 853 \\
34.50 & 854 \\
33.00 & 855 \\
31.50 & 856 \\
30.00 & 857 \\
28.10 & 858 \\
26.70 & 859 \\
25.50 & 860 \\
25.00 & 861 \\
23.70 & 862 \\
\hline
\end{tabular}

Fuente: Elaboración propia

\section{Análisis de los datos y Resultados}

Los datos obtenidos en el laboratorio fueron ampliados en los siguientes aspectos:

- Se obtuvo una columna de datos de temperatura Kelvin del aceite lubricante.

- Se obtuvo también una columna de las densidades corregidas por el efecto térmico sobre el volumen del areómetro, efectuado empleando la ecuación (7).

En la tabla 2, se muestran estos datos ampliados.

Tabla 2: Obtención de datos ampliados y corregidos por el efecto de temperatura sobre el volumen del areómetro

\begin{tabular}{|c|c|c|c|}
\hline $\begin{array}{c}\mathrm{T} \\
\left({ }^{\circ} \mathrm{C}\right)\end{array}$ & $\begin{array}{c}T \\
(K)\end{array}$ & $\left(\mathrm{Kg} / \mathrm{m}^{3}\right)$ & $\begin{array}{c}\rho_{\text {corr }} \\
\left(\mathrm{Kg} / \mathrm{m}^{3}\right)\end{array}$ \\
\hline 45.00 & 318.15 & 848 & 847.7965 \\
\hline 42.00 & 315.15 & 850 & 849.8205 \\
\hline 39.50 & 312.65 & 851 & 850.8407 \\
\hline 37.00 & 310.15 & 852 & 851.8610 \\
\hline 36.00 & 309.15 & 853 & 852.8690 \\
\hline 34.50 & 307.65 & 854 & 853.8811 \\
\hline 33.00 & 306.15 & 855 & 854.8933 \\
\hline 31.50 & 304.65 & 856 & 855.9055 \\
\hline 30.00 & 303.15 & 857 & 856.9177 \\
\hline 28.10 & 301.25 & 858 & 857.9333 \\
\hline 26.70 & 299.85 & 859 & 858.9448 \\
\hline 25.50 & 298.65 & 860 & 859.9546 \\
\hline 25.00 & 298.15 & 861 & 860.9587 \\
\hline 23.70 & 296.85 & 862 & 861.9694 \\
\hline
\end{tabular}

Temperatura de calibración del areómetro (Tc): 20 $\left({ }^{\circ} \mathrm{C}\right)$. Coeficiente lineal de expansión térmica del vidrio Pyrex $\alpha \mathrm{L}=3.2 \mathrm{e}-6\left({ }^{\circ} \mathrm{C}-1\right)$.

Se obtuvo la gráfica y la correlación empírica potencial

Big Bang Faustiniano 2017; 6(3): 27 - 30 de la densidad corregida en función de la temperatura Kelvin, haciendo uso de la Hoja de cálculo EXCEL.

Figura 3: Gráfica de la densidad del aceite Shell Helix HX7 10W-40, en función de la temperatura

\section{DENSIDAD DEL ACEITE SHELL HX7 10W 40 Vs TEMPERTAURA}

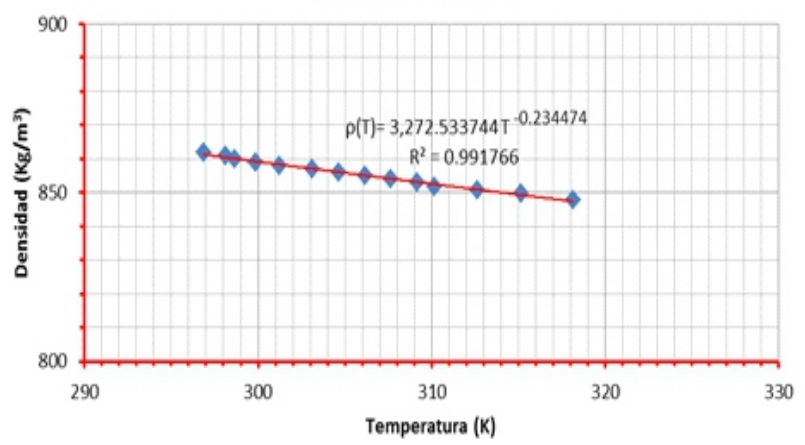

Fuente: Elaboración propia.

\section{CONCLUSIONES}

La correlación obtenida para la densidad del aceite Shell Helix HX7 10W-40, es la siguiente.

$$
\rho(T)=3272,533744(273.15+T)^{-0.234474} \quad\left\{\begin{array}{c}
\rho(T) \rightarrow\left(\frac{K g}{m^{3}}\right) \\
T \rightarrow\left({ }^{\circ} \mathrm{C}\right)
\end{array}\right\}
$$

El coeficiente de correlación de la ecuación empírica es $R^{2}=0.991766$

Para la temperatura $\mathrm{T}=4\left({ }^{\circ} \mathrm{C}\right)$, se obtuvo una densidad $\rho(T)=875.25\left(\mathrm{Kg} / \mathrm{m}^{3}\right)$.

Para la temperatura $\mathrm{T}=100\left({ }^{\circ} \mathrm{C}\right)$, se obtuvo una densidad $\rho(T)=816.29\left(\mathrm{Kg} / \mathrm{m}^{3}\right)$.

Se observa que tiene un buen comportamiento de la densidad en el rango desde 0 a $100\left({ }^{\circ} \mathrm{C}\right)$, para su uso como líquido manométrico del micro manómetro de un tubo de Pitot.

\section{REFERENCIAS}

Benjumea, P., Chávez, G., Vargas, C. (2006). Efecto de la temperatura sobre la densidad de biodiesel de aceite de palma y sus mezclas con diesel convencional. Revista energética 36 (Diciembre del 2006). Universidad Nacional de Colombia.

Wikipedia (9 de Diciembre del 2017). Densímetro.Disponible en: https://es.wikipedia.org/wiki/Dens\%C3\%ADmetro.

Wikipedia (10 de Diciembre del 2017). Coeficiente de dilatación térmica. Disponible en:

https://es.wikipedia.org/wiki/Coeficiente_de_dilataci\% C3\%B3n 


\section{INTRODUCCIÓN}

La etapa formativa en una especialidad médica en Perú es de gran esfuerzo y sacrificio y $90 \%$ es el medico joven que experimenta muchos retos, cambiando sus roles y comenzar a asumir funciones bajo la tutoría de un médico especialista esperando que al cabo de su etapa formativa que puede durar de 3 a 5 años asuma un rol psicobiosocial de manera integral con humanismo y vocación de servicio. Pero dentro de este periodo formativo enfrenta múltiples retos y se encuentran las relaciones intrahospitalarias. En estas relaciones se han identificado maltratos hacia el residente que interfieren con su aprendizaje no solo en Perú si no en todo el mundo. Sin embargo, se conoce poco sobre este fenómeno en Perú o es que no se quiere asumir una posición crítica y correctiva por parte de los Tutores, Presidentes de Especialidad Directores de Post Grado, Decanos y Rectores de manera frontal por perjuicios por tanto estos cargos también son parte del maltrato si es que no se han dado cuenta porque los maltratadores son pocos y al amparo probablemente de las autoridades de forma tácita o explicita pero su daño a los residentes marca una etapa nefasta que se debe erradicar no solo en la labor del profesional asistencial que realiza estas prácticas, si no en la parte académica y social al maltratador y no exista impunidad ante tales hechos.

REVISIÓN DEL PROBLEMA EN PUBLICACIONES MEDICAS INTERNACIONALES

Se ha revisado trabajos de análisis ético y bioético mediante la medición de la percepción de diferentes tipos de maltrato en residentes en todas las publicaciones se observa en promedio de $10 \%$ que ocurren o como se está ocurriendo en nuestro país. Se ha realizado una revisión sistemática de documentos de sociedades científicas que abordan la formación médica. También se han consultado revisiones sistemáticas y estudios científicos sobre el tema a tratarlas cuales son escasas por tan delicado tema a tratar.

Las interrogantes a plantear serian nuestra realidad serían:

1. Cuál es la incidencia del maltrato a los medico Residentes

2. Cuál es la prevalencia del maltrato a los medico Residentes

3. Que especialidad es la que mayor frecuencia se presenta

4. En qué año de formación es mayor su incidencia y prevalencia

5. En cuál de los sistemas públicos ocurre con mayor frecuencia

6. Los Comité Hospitalario tiene conocimiento del maltrato a los residentes

7. La Unidad de Post Grado de las Universidades y diversos funcionarios tiene conocimiento del maltrato a sus residentes.
8. Que acciones se ha realizado para evitar a nivel Académico, Asistencial y social con el maltratador para que no haya impunidad con sus prácticas.

9. Se Identificando los diversos tipos de maltrato, Psicológico, académico, sexual, discriminación.

10. Cuáles son sus consecuencias a corto, mediano y largo plazo que se podrían evitar no solo para el profesional en formación si no para no poner en riesgo a los pacientes como consecuencia de estos hechos.

La revisión me hace trasmitir que en nuestro país se da con frecuencia como ocurre en otros países, pero nadie quiere asumir sus roles o mejor dicho generarse problemas con sus pares que es no correcto de acuerdo al código de ética que tenemos las profesiones médicas y si se denuncia el denunciante es objeto de maltrato por parte de la autoridad en su credibilidad que no debe ser por un falso compañerismo o espíritu de cuerpo que de una manera explícita o tacita se presenta.

Podemos hablar de varios tipos como ejemplo en acoso escolar como un paralelismo en etapa formativa al del Residentado Medico Escolarizado que, a menudo, aparecen de forma simultánea:

Físico: empujones, patadas, agresiones con objetos, etc. Se da con más frecuencia en primaria que en secundaria.

Verbal: insultos y motes, menosprecios en público, resaltar defectos físicos, etc. Es el más habitual.

Psicológico: minan la autoestima del individuo y fomentan su sensación de temor.

Social: pretende aislar al joven del resto del grupo y compañeros.

Estos tres últimos se están dando en el residentado médico.

Es preciso tener en consideración el impacto de "chulear" o "Pimp" o "pimping" es un término inglés que se puede traducir por "chulo" o "proxeneta". Sin embargo, desde la aparición en JAMA en 1989 del artículo de Frederick L Brancati titulado "The Art of Pimping" , En castellano se denomina El bullying que es el maltrato físico y/o psicológico deliberado y continuado que recibe persona por parte de otro $u$ otros, que se comportan con él cruelmente con el objetivo de someterlo y asustarlo, con vistas a obtener algún resultado favorable para los acosadores o simplemente a satisfacer la necesidad de agredir y destruir. En 2009, otro artículo de JAMA escrito por Allan S. Detsky actualiza el de Brancati. Este artículo fue publicado diez años después de que el muy difundido informe del Instituto de Medicina "Errar es humano" apareciese, poniendo de relieve el impacto pernicioso en medicina de la cultura de la vergüenza y la culpa. Pero no es entre niños si no entre personas adultas y lo grave en salud a médicos, no saben que está cometiendo ilícitos Éticos y Bioéticos, Administrativos y me atrevo a decir Penales este último porque alterar el estado psicológico del médico 
residente los expone a cometer errores y por lo tanto la Seguridad se ve vulnerada en la Seguridad del Paciente y la práctica interprofesional.

Desde hace tiempo sabemos que la seguridad del paciente depende de la voluntad de los profesionales a admitir sus errores por lo que podemos, y otros pueden, aprender de ellos. Los pacientes nunca estarán a salvo si los médicos no reconocen lo que no saben, piden ayuda cuando la necesitan, y alertan -en particular a un médico superior- cuando ven a alguien hacer algo malo, peligroso, o imprudente. El Médico residente está formándose en una Especialidad y por tanto su responsabilidad recae en la formación Medica inicial como Medico - Cirujano, título que la Universidad le otorga a nombre de la nación y es mal llamado médico general. Recién su etapa formativa para a una etapa sumativa en su desarrollo profesional en este caso como Médico Especialista.

Si hemos aprendido que una habilidad de comunicación fundamental en la formación médica es comunicar de manera reflexiva y confidencial que no sabemos algo, no expresar nuestras dudas y evitar a toda costa molestar a un superior, hace que la seguridad del paciente llega a ser vulnerada y verdaderamente una misión imposible. Si los médicos aprenden a reverenciar a una jerarquía médica, cuando estos médicos alcancen una respetable antigüedad, pueden interpretar las preocupaciones expresadas de forma auténtica por otros profesionales como retos inaceptables para su estado y su autoridad lo cual es un Dilema Ético y no lo afrontan como tal porque la mayoría en su etapa formativa no han dilucidado o aprendido correctamente. Después de haber usado el "bullying" durante su formación como médicos, estos van a estar predispuestos a "bullying's" también o a rechazar cualquier tipo de autoevaluación crítica, tan esencial en un auténtico y eficiente trabajo clínico de equipo.

Por último, debemos también de tener en cuenta el impacto del "chuleo", "pimping" y/o "bullying" en la relación médico-paciente. Seguramente la mayoría de nosotros, especialmente los que tienen más experiencia como médicos y pacientes a la vez hemos visto y experimentado personalmente cómo algunos médicos -quizás demasiados-, responden a alguna pregunta o comentario difícil. Podemos imaginar bien cómo se puede sentir un paciente al ver como un médico humilla al residente o estudiante que le atiende, es muy probable que este paciente no se atreviese nunca a reclamar por su atención o hacer algo que pueda disgustar a ese médico a cargo de su caso y si aquel es jefe de Servicio o Director del hospital peor aún. Muchos pacientes declaran que les hubiera gustado encontrarse con un médico que simplemente les dijese: "No sé lo que te está pasando, pero vamos a tratar de resolver esto juntos", o que les hubiese pedido disculpas cuando cometieron un error.

Lo que es sorprendente en nuestro medio es que algunos médicos, aunque no de forma y en número suficiente, respondan de esta manera. Si aceptamos la práctica del chuleo y/o bullying, lo que tampoco entonces debería sorprendernos es que algunos médicos no sólo "chuleen", "pimping" y/o "bullying" a los alumnos, sino que también "chuleen" y/o "bullying" a sus pacientes de forma pasiva o activa.

Cada vez en más lugares, especialmente en los Estados Unidos, el acoso y la humillación a la que están sometidos muchos alumnos y residentes empieza a comentarse. Suzzane Gordon, una reputada periodista sanitaria comentaba que en una conferencia médica compartió mesa con una internista conocida defensora de un sistema médico más humano y un médico que trabajaba en la seguridad del paciente. Ambos trabajaban en hospitales docentes. La conversación derivó al "pimping" y la internista comentó que no podía imaginar la enseñanza de los residentes sin en ciertas ocasiones "chulearles". Teniendo en cuenta su reputación, Gordon no podía imaginar que la internista hablaba en serio, y le preguntó si su respuesta era sarcástica. No, le dijo la internista bruscamente, "creo que es una estrategia útil". Entonces el médico de la seguridad del paciente intervino diciéndole: "No, no hay ninguna excusa para que los estudiantes o residentes sean humillados. Absolutamente ninguna" .

El relato anterior no dista de nuestra realidad he sido parte de la indignación de Médicos Residentes de la Especialidad de Anestesia, Analgesia y Reanimación por el simple hecho que el medico jefe de Servicio la solución a la falta de personal de médicos asistentes cumplir su programación quirúrgica desconociendo los convenios de cumplimiento entre la Universidad, Ministerio de Salud y EsSalud. Este caso se dio en un Instituto del MINSA fue solucionado por la intervención directa del Comité de la Especialidad año 2014.

Ahora un nuevo caso en un hospital del Ministerio de Defensa nos toca ser testigo de excepción al por coincidencias del destino una madre de un Médico Residente de la Especialidad de Medicina Intensiva acude a la facultad a buscar ayuda para su hijo por el maltrato verbal, psicológico y social que está pasando y no por un profesional de carrera militar, si no de servicio en otras palabras otro médico que no es y no debe ser permitido por ninguna autoridad y menos hacer espíritu de cuerpo contra el maltratador y dejarlo en la impunidad.

Ahora el 2017 otro caso en un hospital del Ministerio de Salud en la cual en un programa periodístico se propala un hecho de acoso y maltrato a una Médico Residente de Cirugía.

Todo lo relatado pueden ser hechos aislados en la revisión se observa que existe subregistros o no se comunica o hace público por los perjuicios que deviene de estos hechos tanto para el agresor (a), el agredido (a), la institución y el Sistema Público y/o Privado en General. Lo Concreto en otros países donde existe un mejor registro de estos hechos seda entre un $8 \%$ a $10 \%$ probablemente este porcentaje sea mayor en nuestro país. 
La carrera de Medicina nos hace pasar de la adolescencia a la adultez si bien somos mayores de edad y responsables de nuestros actos como ciudadanos pero como transcurre nuestra vida durante ese tiempo no dejamos de estudiar fuerte y cumplir el propósito de ser médicos y no dejamos de ser niños en lo más profundo de nuestro ser y esos actos y actitudes por la impotencia que genera este tipo de conductas por parte de los que son nuestros superiores o posición en la jerarquía dañan como cualquier criminal de guerra que menoscaba la autoestima del enemigo, cuando no somos enemigos si no serán aquellos jóvenes que mañana más tarde nos atenderán o atenderán a nuestras familias y que deseamos que sea los mejores en capacidad, competencia y profesionalismo con un fin supremo respetando la dignidad de las personas en nuestro caso también en la Seguridad de los Pacientes.

Debate: ¿Ha sometido usted en alguna ocasión a "chuleen", "pimping" y/o "bullying" a sus estudiantes, residentes o pacientes?

\section{RECOMENDACIONES}

Los convenios que suscriben las Universidades y las Entidades Prestadoras de Salud que brindan el campo Clínico Publico / Privado, en la formación de Pre Grado (internado) y Post Grado (Residentado) deben constar de manera explícita la prohibición de prácticas que atenten contra la formación del estudiante (interno) y Especialista (Médico Residente) en todas sus modalidades y formas porque no solo atentan con dicho ser humano si no contra todo principio desde la constitución de nuestro país que la dignidad del ser humano está ante todo y sobre todo y afecta a tercera personas que son los pacientes en otras palabras la Seguridad del Paciente.

\section{Sumando a ello.}

1. Se necesita realizar estudios para conocer la realidad de dichas prácticas en los profesionales en formación internos y residentes.

2. Se necesita realizar estudios para conocer la realidad de dichas prácticas en los pacientes.

3. Se requiere implementar planes de trabajo destinados a optimizar los procesos operativos de docencia e investigación (de contacto directo con el usuario) y de soporte (de apoyo a la ejecución de los procesos formativos) que se realizan en los servicios, para garantizar la calidad de la atención y seguridad tanto de los pacientes como del personal de salud y administrativo, debiéndose priorizar los recursos necesarios.

\section{CONCLUSIONES}

Contrastando la información de las publicaciones médicas internacionales. Se cuenta con estudios, pero no son datos oficiales sobre "chuleen", "pimping" y/o "bullying" a sus estudiantes, residentes o pacientes en los hospitales, así como tampoco tenemos publicaciones sobre soluciones para tratar "chuleen", "pimping" y/o "bullying". Tenemos en mano un tema tan importante como desafío ante para investigación que interesa sobre manera a los principales formadores y prestadores de servicios públicos de salud. Tienen un compromiso con la sociedad en sincerar públicamente ese como otros problemas de gestión, a fi $n$ de enfocar las más adecuadas soluciones a corto y mediano plazo con los recursos disponibles, con metas en la mejora continua de la calidad de atención de los pacientes, esto último expresado como la cuarta etapa de la calidad que no es otra cosa que la seguridad del Paciente. así como las condiciones ambientes de trabajo en la etapa pre profesional (internos) y profesional (médicos residentes) o mejor dicho de cualquier estudiante de las profesiones de salud en su etapa formativa, no debe existir estas prácticas y si existen estas prácticas no debe quedar impune. en razón de cumplir con la obligación como ciudadanos que nos demanda nuestra constitución y se consagra en el Artículo $1^{\circ}$. - La defensa de la persona humana y el respeto de su dignidad son el fin supremo de la sociedad y del Estado.

\section{REFERENCIAS BIBLIOGRÁFICAS}

1.FL B. The art of pimping. 1989 jul; 7(262 (1)): p. 8990.

2. AS D. The art of pimping. 2009 Apr; 1 (301 (13)): p. 1379-81.

3. Medicine lo. To Err is Human: Building A Safer Health Systen. 1989.

4. S. G. Pimping has no place in medical educatión. BMJ. 2015April; 15.

5. http//blogs.bmj.com/2015/04/15/suzane-gordonpimping-has-no-place-in-medical-educatio/. 